PROCEEDINGS OF THE AMERICAN MATHEMATICAL SOCIETY

Volume 124, Number 11, November 1996

\title{
COMBINATORICS OF A CERTAIN IDEAL IN THE SEGRE COORDINATE RING
}

\author{
PAULO BRUMATTI, PHILIPPE GIMENEZ, AND ARON SIMIS
}

(Communicated by Wolmer V. Vasconcelos)

\begin{abstract}
We focus on a "fat" model of an ideal in the class of the canonical ideal of the Segre coordinate ring, looking at its Rees algebra and related arithmetical questions.
\end{abstract}

\section{INTRODUCTION}

Let $\mathfrak{S}$ be the image of the Segre map

$$
\sigma=\sigma_{n-1, m-1}: \mathbb{P}^{n-1} \times \mathbb{P}^{m-1} \longrightarrow \mathbb{P}^{n m-1},
$$

the so-called Segre variety. As a toric variety, $\mathfrak{S}$ admits $k\left[t_{i} s_{j}\right](1 \leq i \leq n, 1 \leq$ $j \leq m)$ as coordinate ring. This ring can be presented over the polynomial ring $k[\mathbf{X}]=k\left[X_{i j}\right](1 \leq i \leq n, 1 \leq j \leq m)$ by the ideal $I_{2}\left(X_{i j}\right)$ generated by the $2 \times 2$ minors of the generic $n \times m$ matrix $\left(X_{i j}\right)$. It is well known that the canonical class of the latter is $(m-n)[\mathfrak{K}]$, where $\mathfrak{K} \subset S=k[\mathbf{X}] / I_{2}\left(X_{i j}\right)$ is the ideal generated by (the residues of) the entries in the first column of the matrix $\left(X_{i j}\right)$ (cf. [BV], (8.4)).

Now, given an integer $d \geq 1$, let $\mathfrak{K}^{[d]}$ denote the ideal generated by the $d$ th powers of the generators of $\mathfrak{K}$. The main purpose of this paper is to investigate the algebraic-combinatorics of the blowup of $\mathfrak{S}$ along the locus of $\mathfrak{K}^{[d]}$. Algebraically, we are therefore looking at the Rees algebra of the ideal $\mathfrak{K}^{[d]}$. Using the toric representation, this algebra is simply the $k$-subalgebra

$$
k\left[t_{i} s_{j},\left(t_{1} s_{1}\right)^{d} T, \ldots,\left(t_{n} s_{1}\right)^{d} T\right] \subset k[\mathbf{t}, \mathbf{s}][T],
$$

where $1 \leq i \leq n, 1 \leq j \leq m$. Since $s_{1}$ is fixed in the $d$ th powers, it is not difficult to see that this algebra is isomorphic to the $k$-algebra $R^{[d]}=k\left[t_{i} s_{j}, t_{1}^{d}, \ldots, t_{n}^{d}\right] \subset$ $k[\mathbf{t}, \mathbf{s}]$.

As it turns out, $R^{[d]}$ is presented over a polynomial $\operatorname{ring} A=k[\mathbf{X}, \mathbf{U}]$, with $\mathbf{X}=\left\{X_{i j}\right\}, \mathbf{U}=\left\{U_{1}, \ldots, U_{n}\right\}$, by a sum of determinantal ideals, each generated by certain $2 \times 2$ minors, so our toric variety is a sort of determinantal locus lacking the generic codimension. It can be looked at as the generic version of a few classes of ideals appearing in the recent literature (cf. [Hu], [HuHu], [Sch $]$ and $[\mathrm{MoSi}])$, obtained thereof by specialization and by taking suitable free ring extensions.

Received by the editors April 25, 1995.

1991 Mathematics Subject Classification. Primary 13H10; Secondary 13C05, 13H15, 13P10.

Key words and phrases. Segre ring, monomials, Rees algebra, Cohen-Macaulay, polarization.

The first and the third authors were partially supported by CNPq.

The second author is grateful for the warm hospitality during his visit to Brazilian institutions. He thanks UniCamp and CDE (IMU) for providing resources for this visit. 


\section{A PSEUDO-DETERMINANTAL LOCUS}

We will fix the following notation:

$I_{r}(L) \quad$ the ideal generated by $r \times r$ minors of the matrix $L$.

$\mathbf{t}, \mathbf{s} \quad$ sets of (toric) variables $t_{1}, \ldots, t_{n}, s_{1}, \ldots, s_{m}$ over a field $k$.

$S \quad$ the coordinate ring $k\left[x_{i j}\right]=k\left[X_{i j}\right] / I_{2}\left(X_{i j}\right)$ of the Segre embedding.

$\mathfrak{K}^{[d]} \quad$ the ideal (row-matrix) in $S$ generated by the $d$ th powers of $x_{11}, \ldots$,

$R^{[d]} \quad$ the toric $\operatorname{ring} k\left[t_{i} s_{j}, t_{1}^{d}, \ldots, t_{n}^{d}\right]$.

$M(\mathbf{Y}) \quad$ a monomial in the variables $\mathbf{Y}$.

$M(\mathbf{y}) \quad$ the residue of the monomial $M(\mathbf{Y})$ modulo some ideal.

$\mathbb{M}(d, \mathbf{Y})$ the set (row, ideal) of all monomials of degree $d$ in the variables $\mathbf{Y}$.

$\mathbb{M}(d, \mathbf{y}) \quad$ the set of residues of $\mathbb{M}(d, \mathbf{Y})$.

2.1. The defining equations. One needs the following lemmata. In order to save on notation, we set sometimes $\mathbf{X}_{i}=X_{i 1}, \ldots, X_{i m}$ and, correspondingly, $\mathbf{x}_{i}=$ $x_{i 1}, \ldots, x_{i m}$.

(2.1.1) Lemma. For any pair of indices $1 \leq i_{1}, i_{2} \leq n$, consider the involutive $k$-algebra automorphism $\Phi=\Phi_{i_{1}, i_{2}}$ of the polynomial ring $k\left[X_{i j}\right]=k\left[\mathbf{X}_{1}, \ldots, \mathbf{X}_{n}\right]$ such that

$$
\Phi\left(X_{i, j}\right)= \begin{cases}X_{i_{2}, j} & \text { if } i=i_{1} \\ X_{i_{1}, j} & \text { if } i=i_{2} \\ X_{i, j} & \text { otherwise. }\end{cases}
$$

Then:

(i) $\Phi$ induces an automorphism of $S=k\left[X_{i j}\right] / I_{2}\left(X_{i j}\right)$.

(ii) For any two monomials $M=M\left(\mathbf{X}_{i_{1}}\right), N=N\left(\mathbf{X}_{i_{1}}\right) \in k\left[\mathbf{X}_{i_{1}}\right]$ of the same degree, one has $M \Phi_{i_{1}, i_{2}}(N) \equiv N \Phi_{i_{1}, i_{2}}(M)\left(\bmod I_{2}\left(X_{i j}\right)\right)$.

Proof. (i) Clearly, the ideal $I_{2}\left(X_{i j}\right)$ is invariant under $\Phi$. Since $\Phi$ is an involution (i.e., $\Phi=\Phi^{-1}$ ), it then induces an automorphism of $S$.

(ii) One proceeds by induction on the common degree of $M$ and $N$. The result is trivial if $M=N$, so assume these are distinct monomials. Now write $M=X_{i_{1}, j_{1}} M_{1}$ and $N=X_{i_{1}, j_{2}} N_{1}$, with $j_{1} \neq j_{2}$. Then, with $\Phi=\Phi_{i_{1}, i_{2}}$ and by the inductive hypothesis:

$$
\begin{aligned}
M \Phi(N) & =X_{i_{1}, j_{1}} X_{i_{2}, j_{2}} M_{1} \Phi\left(N_{1}\right) \equiv X_{i_{1}, j_{2}} X_{i_{2}, j_{1}} M_{1} \Phi\left(N_{1}\right) \\
& \equiv X_{i_{1}, j_{2}} X_{i_{2}, j_{1}} N_{1} \Phi\left(M_{1}\right)=N \Phi(M)
\end{aligned}
$$

as required.

(2.1.2) Remark. Part (ii) of Lemma (2.1.1) has been used before in different forms (cf., e.g., [Gim, Lemme 5.12.1]).

(2.1.3) Lemma. The first syzygies of the ideal $\mathfrak{K}^{[d]} \subset S$ are generated by the first syzygies of all pairs $\left\{x_{i_{1}, 1}^{d}, x_{i_{2}, 1}^{d}\right\}, 1 \leq i_{1}, i_{2} \leq n$ and these are generated by those syzygies whose coordinates are terms $\alpha M, \alpha \in k$ and $M$ a monomial.

Proof. This is a direct consequence of the fact that $S$ is defined by a binomial ideal [EiSt, Corollary 1.7 (b)]. 
Here is the basic technical result of this section:

(2.1.4) Proposition. Let $d \geq 1$. The ideal $\mathfrak{K}^{[d]} \subset S$ has the following presentation as an $S$-module:

$$
\left(\bigwedge^{2} S^{n}\right)^{\oplus^{C(m, d)}} \stackrel{\psi^{[d]}}{\longrightarrow} S^{n} \stackrel{\mathfrak{K}^{[d]}}{\longrightarrow} S,
$$

where $C(m, d)=\left(\begin{array}{c}m-1+d \\ d\end{array}\right), \mathfrak{K}^{[d]}$ stands for the map given by the row-matrix $\left(x_{11}^{d} \ldots x_{n 1}^{d}\right)$ and $\psi^{[d]}$ is given by the matrix

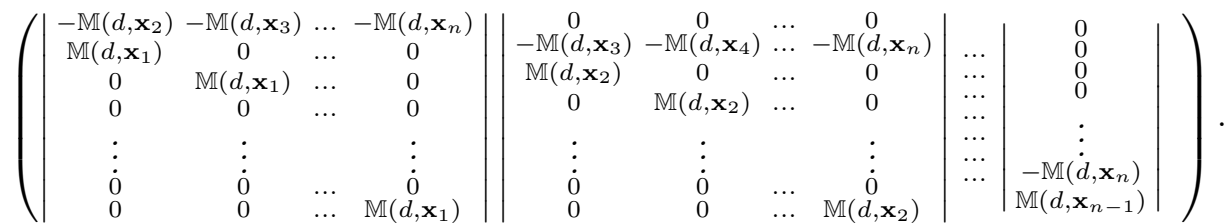

Proof. The containment $\operatorname{Im} \psi^{[d]} \subset \operatorname{ker} \mathfrak{K}^{[d]}$ is a straightforward consequence of Lemma (2.1.1), (ii).

For the reverse inclusion, by Lemma (2.1.3) and by an obvious symmetrical argument we may assume that we are given a relation of the form

$$
X_{11}^{d} M+X_{21}^{d} N \equiv 0 \quad\left(\bmod I_{2}\left(X_{i j}\right)\right),
$$

where $M$ and $N$ are terms in $k[\mathbf{X}]$.

The crucial point is to establish that $\operatorname{deg}_{\mathbf{X}_{2}} M \geq d$. At any rate, one has $\operatorname{deg}_{\mathbf{X}_{2}} M \geq 1$, otherwise by setting to zero all the variables in $\mathbf{X}_{2}$, it would follow that $X_{11}^{d} M \in I_{2}\left(X_{i j}\right)(i \neq 2)$ which is absurd.

We proceed by induction on $d$, the assertion for $d=1$ having just been shown. Thus, let $d \geq 2$ and assume that $\operatorname{deg}_{\mathbf{X}_{2}} M=d_{0}<d$. By the preceding, $d_{0} \geq 1$, hence $d-d_{0} \leq d-1$. Write $M=\widetilde{M} M_{1}$, with $\widetilde{M} \in \mathbb{M}\left(d_{0}, \mathbf{X}_{2}\right)$. By Lemma (2.1.1), (ii), one has $X_{11}^{d_{0}} \widetilde{M} \equiv X_{21}^{d_{0}} \Phi_{12}(\widetilde{M})$, hence (2-1) yields

$$
-X_{21}^{d} N \equiv X_{11}^{d_{0}} \cdot X_{11}^{d-d_{0}} \widetilde{M} M_{1} \equiv X_{21}^{d_{0}} \cdot X_{11}^{d-d_{0}} \Phi_{12}(\widetilde{M}) M_{1}
$$

from which it follows that $X_{11}^{d-d_{0}} \Phi_{12}(\widetilde{M}) M_{1}+X_{21}^{d-d_{0}} N \equiv 0$. Then, by the inductive hypothesis we know that $\operatorname{deg}_{\mathbf{X}_{2}} \Phi_{12}(\widetilde{M}) M_{1} \geq d-d_{0}>0$. But since $\Phi_{12}(\widetilde{M}) \in$ $\mathbb{M}\left(d_{0}, \mathbf{X}_{1}\right)$, we see that $\operatorname{deg}_{\mathbf{X}_{2}} \Phi_{12}(\widetilde{M}) M_{1}=\operatorname{deg}_{\mathbf{X}_{2}} M_{1}=0$, a contradiction.

Thus, we can write $M=\widetilde{M} M_{1}$, where $\widetilde{M} \in \mathbb{M}\left(d, \mathbf{X}_{2}\right)$. By Lemma (2.1.1), (ii), we have $X_{11}^{d} \widetilde{M} \equiv X_{21}^{d} \Phi_{12}(\widetilde{M})$, from which it follows that $X_{11}^{d} M \equiv X_{21}^{d} \Phi_{12}(\widetilde{M}) M_{1}$. Using (2-1), one then obtains $X_{21}^{d}\left(\Phi_{12}(\widetilde{M}) M_{1}+N\right) \equiv 0$, hence $N \equiv-\Phi_{12}(\widetilde{M}) M_{1}$ because $I_{2}\left(X_{i j}\right)$ is a prime ideal. Since $\widetilde{M} \in \mathbb{M}\left(d, \mathbf{X}_{2}\right)$, it follows that $\Phi_{12}(\widetilde{M}) \in$ $\mathbb{M}\left(d, \mathbf{X}_{1}\right)$.

Altogether, one gets

$$
\left(\begin{array}{c}
M(\mathbf{x}) \\
N(\mathbf{x})
\end{array}\right)=M_{1}(\mathbf{x})\left(\begin{array}{c}
\widetilde{M}\left(\mathbf{x}_{2}\right) \\
-\left(\widetilde{M}\left(\mathbf{x}_{1}\right)\right)
\end{array}\right) \in \operatorname{Im} \psi^{[d]},
$$

as was to be shown.

Here is the main result of this section.

(2.1.5) Theorem. Let $d \geq 1$ be an integer and let $\mathfrak{K}^{[d]} \subset S=k\left[X_{i j}\right] / I_{2}\left(X_{i j}\right)$ as before stand for the ideal generated by the dth powers of the generators of the ideal 
$\mathfrak{K}$ of $S$. Also let $R^{[d]}=k\left[t_{i} s_{j}, t_{1}^{d}, \ldots, t_{n}^{d}\right] \subset k[\mathbf{t}, \mathbf{s}](1 \leq i \leq n, 1 \leq j \leq m)$. Then:

(i) The ideal $\mathfrak{K}^{[d]}$ is of linear type.

(ii) There is a presentation

$$
R^{[d]} \simeq k\left[\mathbf{X}_{1}, \mathbf{X}_{2}, \ldots, \mathbf{X}_{n}, \mathbf{U}\right] / \sum_{1 \leq i_{1}<i_{2} \leq n} I_{2}\left(L_{i_{1}, i_{2}}\right)
$$

where

$$
L_{i_{1}, i_{2}}=\left(\begin{array}{ll}
X_{i_{1}, 1} \ldots X_{i_{1}, m} & U_{i_{1}} \cdot \mathbb{M}\left(d-1, \mathbf{X}_{i_{2}}\right) \\
X_{i_{2}, 1} \ldots X_{i_{2}, m} & U_{i_{2}} \cdot \mathbb{M}\left(d-1, \mathbf{X}_{i_{1}}\right)
\end{array}\right),
$$

with $U_{i_{l}} \cdot \mathbb{M}\left(d-1, \mathbf{X}_{i_{l}}\right)$ designating the row whose entries are the entries of $\mathbb{M}\left(d-1, \mathbf{X}_{i_{l}}\right)$ multiplied by the variable $U_{i_{l}}$.

Proof. (i) We show that the generators $x_{11}^{d}, \ldots, x_{n 1}^{d}$ of $\mathfrak{K}^{[d]}$ form a $d$-sequence. For that, we use the characterization of such sequences as given in [HSV, Section 6] to the effect that

$$
\left(\left(x_{11}^{d}, \ldots, x_{s 1}^{d}\right): x_{s+11}^{d}\right) \cap \mathfrak{K}^{[d]}=\left(x_{11}^{d}, \ldots, x_{s 1}^{d}\right) \quad \text { for } 0 \leq s \leq n-1 .
$$

By Proposition (2.1.4), one sees that

$$
\left(\left(x_{11}^{d}, \ldots, x_{s 1}^{d}\right): x_{s+11}^{d}\right)=\left(\mathbb{M}\left(d, \mathbf{x}_{1}\right), \mathbb{M}\left(d, \mathbf{x}_{2}\right), \ldots, \mathbb{M}\left(d, \mathbf{x}_{s}\right)\right),
$$

hence we are to prove that

$$
\left(\mathbb{M}\left(d, \mathbf{x}_{1}\right), \mathbb{M}\left(d, \mathbf{x}_{2}\right), \ldots, \mathbb{M}\left(d, \mathbf{x}_{s}\right)\right) \cap\left(x_{11}^{d}, \ldots, x_{n 1}^{d}\right) \subset\left(x_{11}^{d}, \ldots, x_{s 1}^{d}\right) .
$$

Set $J_{1}=\left(\mathbb{M}\left(d, \mathbf{x}_{1}\right), \mathbb{M}\left(d, \mathbf{x}_{2}\right), \ldots, \mathbb{M}\left(d, \mathbf{x}_{s}\right)\right)$ and $J_{2}=\left(x_{11}^{d}, \ldots, x_{n 1}^{d}\right)$.

To compute the above intersection of monomial ideals modulo the binomial ideal $I_{2}\left(X_{i j}\right)$ we follow the prescription given in [EiSt, Proof of Corollary 1.6]: choose a monomial order on the polynomial ring $A=k\left[X_{i j}\right]$ and take the standard monomials $\bmod I_{2}\left(X_{i j}\right)$; then, $\mathcal{M}(\mathbf{x}) \subset A / I_{2}\left(X_{i j}\right)$, the set of residues of the standard monomials, is a vector space basis of $A / I_{2}\left(X_{i j}\right)$; next, one takes a vector space basis $\mathcal{J}_{1}\left(\operatorname{resp} \mathcal{J}_{2}\right)$ of $J_{1}\left(\right.$ resp. $\left.J_{2}\right) \bmod I_{2}\left(X_{i j}\right)$ which is contained in $\mathcal{M}(\mathbf{x})$; at the outset, $\mathcal{J}_{1} \cap \mathcal{J}_{2}$ is a vector space basis of the ideal $J_{1} \cap J_{2}$.

Now, in the present case, choosing a suitable order, the $2 \times 2$ minors already form a Gröbner basis of the ideal $I_{2}\left(X_{i j}\right)$ (cf., e.g., [Stu]). Therefore, a monomial in $\mathcal{M}(\mathbf{x})$ is characterized by the property that it involves indeterminates belonging to one and only one row or to one and only one column of the matrix $\left(x_{i j}\right)$. It follows from this that

$$
\mathcal{J}_{1}=\left(\bigcup_{\substack{1 \leq i \leq s \\ r \geq d}} \mathbb{M}\left(r, \mathbf{x}_{i}\right)\right) \cup\left\{x_{i j}^{d} M\left(\mathbf{x}^{j}\right) \mid 1 \leq i \leq s, 1 \leq j \leq m\right\}
$$

is a vector basis of $J_{1}$, where $M\left(\mathbf{x}^{j}\right)$ designates a monomial involving only variables along the $j$ th column.

By a similar token,

$$
\mathcal{J}_{2}=\left\{x_{i 1}^{d} M\left(\mathbf{x}^{j}\right), x_{i 1}^{d} M\left(\mathbf{x}_{i}\right) \mid 1 \leq i \leq n\right\}
$$

is a vector basis of $J_{2}$, where $M\left(\mathbf{x}_{i}\right)$ designates a monomial involving only variables along the $i$ th row. One clearly has $\mathcal{J}_{1} \cap \mathcal{J}_{2}=\left\{x_{i 1}^{d} M\left(\mathbf{x}^{j}\right), x_{i 1}^{d} M\left(\mathbf{x}_{i}\right) \mid 1 \leq i \leq s\right\}$. Therefore, the ideal $J_{1} \cap J_{2}$ is generated by $\left\{x_{i 1}^{d}, \mid 1 \leq i \leq s\right\}$, as was to be shown. 
(ii) By part (i), the canonical surjection $\mathcal{S}\left(\mathfrak{K}^{[d]}\right) \rightarrow \mathcal{R}\left(\mathfrak{K}^{[d]}\right)$ is an isomorphism, where $\mathcal{S}\left(\mathfrak{K}^{[d]}\right)$ and $\mathcal{R}\left(\mathfrak{K}^{[d]}\right)$ denote the symmetric and the Rees algebra of the ideal $\mathfrak{K}^{[d]}$, respectively. On the other hand, by Proposition (2.1.4), $\mathcal{S}\left(\mathfrak{K}^{[d]}\right)$ admits the presentation that is being proposed for $R^{[d]}$. Therefore, it suffices to show that $R^{[d]}$ is isomorphic to $\mathcal{R}\left(\mathfrak{K}^{[d]}\right)$. Clearly,

$$
\mathcal{R}\left(\mathfrak{K}^{[d]}\right) \simeq S\left[\mathfrak{K}^{[d]} T\right] \simeq k\left[t_{i} s_{j},\left(t_{1} s_{1}\right)^{d} T, \ldots,\left(t_{n} s_{1}\right)^{d} T\right] \subset k[\mathbf{t}, \mathbf{s}][T] .
$$

Since $s_{1}^{d}$ is a common factor throughout the terms $t_{i}^{d} s_{1}^{d} T$ and these have a fixed degree, we see that there is an isomorphism $k\left[t_{i} s_{j}, t_{1}^{d}, \ldots, t_{n}^{d}\right] \simeq k\left[t_{i} s_{j},\left(t_{1} s_{1}\right)^{d} T, \ldots\right.$, $\left.\left(t_{n} s_{1}\right)^{d} T\right]$.

2.2. Hilbert function data of $R^{[d]}$. The reader is referred to [HUT] and [STV] for the background needed in this portion. Again, one considers the Segre ring $S=k[\mathbf{X}] / I_{2}(\mathbf{X})$, which will be thought of as the current base ring. By Theorem (2.1.5), $R^{[d]}$ is isomorphic to the Rees algebra of the ideal $\mathfrak{K}^{[d]} \subset S$ and, moreover, as such, it has a natural structure of standard bigraded $k$-algebra, its presentation ideal over $S$ being bihomogeneous with respect to the two sets of variables $\mathbf{X}=\left\{X_{i j}\right\}$ and $\mathbf{U}=\left\{U_{1}, \ldots, U_{n}\right\}$.

Consider an $\mathbb{N}^{n+1}$-gradation on $S[\mathbf{U}]$ by setting

$$
S[\mathbf{U}]_{\left(a_{0}, a_{1}, \ldots, a_{n}\right)}:=S_{a_{0}} U_{1}^{a_{1}} \cdots U_{n}^{a_{n}} .
$$

Let $\succeq$ be the graded lexicographic order on the monoid $\mathbb{N}^{n+1}$. It induces a filtration $\mathcal{F}$ on $S[\mathbf{U}]$, with $\mathcal{F}_{\mathbf{a}}:=\oplus_{\mathbf{b} \succeq \mathbf{a}} S[\mathbf{U}]_{\mathbf{b}}$, hence also on the residue ring $R^{[d]} \simeq S[\mathbf{U}] / \mathcal{J}$ which we still denote by $\mathcal{F}$. Letting $\mathcal{J}^{*}$ denote the ideal generated by the initial forms of $\mathcal{J}$, one has $\operatorname{gr}_{\mathcal{F}}\left(\left(R^{[d]}\right)\right) \simeq S[\mathbf{U}] / \mathcal{J}^{*}$ as bigraded $k$-algebras.

By Proposition (2.1.4) (or by the proof of Theorem (2.1.5), (i)) and [HUT, Lemma 1.1], one obtains

$$
\mathcal{J}^{*}=\left(\mathbb{M}\left(d, \mathbf{x}_{1}\right) U_{2},\left(\mathbb{M}\left(d, \mathbf{x}_{1}\right), \mathbb{M}\left(d, \mathbf{x}_{2}\right)\right) U_{3}, \ldots,\left(\mathbb{M}\left(d, \mathbf{x}_{1}\right), \ldots, \mathbb{M}\left(d, \mathbf{x}_{n-1}\right)\right) U_{n}\right) .
$$

(2.2.1) Proposition. With the preceding notation and considering $R^{[d]}$ and $\operatorname{gr}_{\mathcal{F}}\left(\left(R^{[d]}\right)\right.$ ) as $\mathbb{N}$-graded rings (via the homomorphism $\mathbb{N}^{2} \rightarrow \mathbb{N},(a, b) \mapsto a+b$ ), one has:

(i) $R^{[d]}$ and $\operatorname{gr}_{\mathcal{F}}\left(\left(R^{[d]}\right)\right)$ admit the same Hilbert function.

(ii) The multiplicity of $R^{[d]}$ is

$$
e\left(R^{[d]}\right)=\sum_{j=0}^{n-1} d^{j}\left(\begin{array}{c}
m+n-j-2 \\
n-j-1
\end{array}\right) .
$$

Proof. (i) This is easy and holds quite generally.

(ii) We apply [HUT, Theorem 1.4] (or rather, its recipe), for which we first check its hypotheses. In the present situation, they boil down to the equalities

$$
\operatorname{dim} S / I_{j}=\operatorname{dim} S-j, \quad 1 \leq j \leq n-1,
$$

where $I_{j}=\left(\mathbb{M}\left(d, \mathbf{x}_{1}\right), \ldots, \mathbb{M}\left(d, \mathbf{x}_{j}\right)\right)$. To verify these, we show that ht $I_{j}=j$ for $1 \leq j \leq n-1$ (recalling that $S$ is Cohen-Macaulay). For every such $j$, consider the prime ideal

$$
\begin{aligned}
P_{j} & =\left(\left\{X_{k l} \mid 1 \leq k \leq j, 1 \leq l \leq m\right\}\right)+I_{2}\left(\left\{X_{k^{\prime} l} \mid j+1 \leq k^{\prime} \leq n, 1 \leq l \leq m\right\}\right) \\
& =\left(\left\{\mathbf{X}_{1}, \ldots, \mathbf{X}_{j}\right\}\right)+I_{2}\left(\mathbf{X} \backslash\left\{\mathbf{X}_{1}, \ldots, \mathbf{X}_{j}\right\}\right) \subset k[\mathbf{X}] .
\end{aligned}
$$


Clearly, $P_{j} S$ is a prime as well and contains $I_{j}$. It follows that

$$
\text { ht } \begin{aligned}
I_{j} & \leq \operatorname{ht} P_{j} S=\operatorname{ht} P_{j}-\operatorname{ht} I_{2}(\mathbf{X}) \\
& =\operatorname{ht}\left(\left\{\mathbf{X}_{1}, \ldots, \mathbf{X}_{j}\right\}\right)+\operatorname{ht} I_{2}\left(\mathbf{X} \backslash\left\{\mathbf{X}_{1}, \ldots, \mathbf{X}_{j}\right\}\right)-(n-1)(m-1) \\
& =j m+(n-j-1)(m-1)-(n-1)(m-1)=j
\end{aligned}
$$

On the other hand, it is easy to see that every prime ideal of $S$ containing $I_{j}$ already contains $P_{j} S$. This leads to ht $I_{j}=j$, as required.

We now compute the multiplicity $e\left(S / I_{j}\right)$ by the associativity formula. By the above calculation, this formula reduces to

$$
e\left(S / I_{j}\right)=\ell\left(S_{P_{j} S} / I_{j_{j} S}\right) e\left(S / P_{j} S\right) .
$$

To simplify the notation, set $P=P_{j}, I=I_{j}$. Observe that the ideal $P S_{P S} / I_{P S}$ is generated by the images of the variables $X_{11}, \ldots, X_{j 1}$. Indeed, typically, $X_{k 1} X_{n l}-$ $X_{k l} X_{n 1} \equiv 0\left(\bmod I_{2}(\mathbf{X})\right)$. Since $X_{n 1}$ is invertible, the image of $X_{k l}$ belongs to the ideal generated by the image of $X_{k 1}$, for $1 \leq k \leq j$. The above length is then given by the number of monomials $\left\{X_{11}^{a_{1}} \cdots X_{j 1}^{a_{j}} \mid 0 \leq a_{k} \leq d-1,1 \leq k \leq j\right\}$. This number is clearly $d^{j}$.

Next, one has $S / P_{j} S=k[\mathbf{X}] / P \simeq k\left[\mathbf{X} \backslash\left\{\mathbf{X}_{1}, \ldots, \mathbf{X}_{j}\right\}\right] / I_{2}\left(\mathbf{X} \backslash\left\{\mathbf{X}_{1}, \ldots, \mathbf{X}_{j}\right\}\right)$, which is a Segre ring of size $(n-j) \times m$. Therefore, $e(S / P)=\left(\begin{array}{c}m+n-j-2 \\ n-j-1\end{array}\right)$ by a well-known formula (cf., e.g., [STV, Remark 2.5]).

To piece everything together, [HUT, Theorem 1.4] tells us that $e\left(R^{[d]}\right)=$ $\sum_{j=0}^{n-1} e\left(S / I_{j}\right)$, hence we are through.

(2.2.2) Remark. By Proposition (2.2.1), (i), it is in principle possible to compute the Hilbert function of $R^{[d]}$, but it is hardly the case that it may be of any uselfuness here. Thus, for example, $\operatorname{dim} R^{[d]}=m+n$ follows directly from the fact that $R^{[d]}$ is a Rees algebra of an ideal in the $m+n$-1-dimensional domain $S$.

\section{The Defining equations of the SPecial Algebra}

As above, let $I=I^{[d]} \subset k[\mathbf{X}, \mathbf{U}]$ denote the presentation ideal of the $k$-algebra $R^{[d]}$ and let $\tilde{I}=I S[\mathbf{U}] \subset S[\mathbf{U}]$, an ideal generated in bidegree $(d, 1)$. We consider the Rees algebra $\mathcal{R}_{S[\mathbf{U}]}(\tilde{I})$ : geometrically, one is looking at the blowup of the product $\mathfrak{S} \times \mathbb{P}_{\mathbf{U}}^{n-1}$ along the subvariety $\mathcal{B} \ell_{\mathcal{K}}(\mathfrak{S})$, where $\mathcal{K}$ denotes the subvariety of $\mathfrak{S}$ defined by the ideal $\mathfrak{K}^{[d]}$.

The special algebra (or fiber cone algebra) of an ideal (resp. homogeneous ideal) $\mathfrak{a}$ in a local (resp. positively graded) ring $A$ is the residue ring $\mathcal{F}(\mathfrak{a}):=$ $\mathcal{R}_{A}(\mathfrak{a}) / \mathfrak{m} \mathcal{R}_{A}(\mathfrak{a})$, with $\mathfrak{m}$ standing for the maximal (resp. maximal graded) ideal of A.

We will take $A=S[\mathbf{U}]$ and $\mathfrak{a}=\tilde{I}$. As it will turn out, $\mathcal{F}(\tilde{I})$ is a nice determinantal locus which, in the case where $n=2$, is the coordinate ring of a Veronese variety. The reason for that is a far more reaching principle which may have an independent interest outside the scope of the present work.

(3.1) Theorem. Let $\mathbf{X}, \mathbf{Y}$ be mutually independent sets of variables over a field $k$ of characteristic zero, with $\mathbf{X}$ and $\mathbf{Y}$ having the same number of elements, and let $f_{1}, \ldots, f_{r}$ be homogeneous polynomials in the $\mathbf{X}$-variables, of the same degree. Let $U, V$ be two additional variables and set $A=k\left[f_{1} V-\Phi\left(f_{1}\right) U, \ldots, f_{r} V-\Phi\left(f_{r}\right) U\right] \subset$ 
$k[\mathbf{X}, \mathbf{Y}, U, V]$, where $\Phi$ as in Lemma (2.2.1) denotes the involutive $k$-isomorphism $X_{i} \mapsto Y_{i}$. Then

$$
k\left[f_{1}, \ldots, f_{r}\right] \simeq A / A \cap I_{2}(\mathbf{X}, \mathbf{Y}) k[\mathbf{X}, \mathbf{Y}, U, V]
$$

as graded $k$-algebras, where $I_{2}(\mathbf{X}, \mathbf{Y})$ denotes the ideal of $k[\mathbf{X}, \mathbf{Y}]$ generated by the $2 \times 2$ minors of the generic matrix whose rows are $\mathbf{X}$ and $\mathbf{Y}$.

Proof. Let $T_{1}, \ldots, T_{r}$ be presentation variables over $k$ for both algebras. It will suffice to show that they have the same presentation ideal. We show, namely, that any homogeneous polynomial relation of one of the two algebras is a polynomial relation of the other. We need the notion of polarization.

Consider a polynomial ring $k[\mathbf{T}, \mathbf{U}]$ in two sets of indeterminates $\mathbf{T}=T_{1}, \ldots, T_{r}$ and $\mathbf{U}=U_{1}, \ldots, U_{r}$. Clearly, $k[\mathbf{T}, \mathbf{U}]$ is a free $k[\mathbf{U}]$-module with basis the monomials in $\mathbf{T}$.

(3.2) Definition. The polarization of $\mathbf{T}$ by $\mathbf{U}$ is the (unique) $k[\mathbf{U}]$-homomorphism $P$ of the $k[\mathbf{U}]$-module $k[\mathbf{T}, \mathbf{U}]$ such that $P(1)=0$ and

$$
P\left(\mathbf{T}^{a}\right)=\sum_{a_{j} \neq 0} a_{j} U_{j} T_{1}^{a_{1}} \cdots T_{j}^{a_{j}-1} \cdots T_{r}^{a_{r}}
$$

for $\mathbf{T}^{a}=T_{1}^{a_{1}} \cdots T_{r}^{a_{r}}$.

One sets $P_{0}\left(\mathbf{T}^{a}\right)=\mathbf{T}^{a}$ and $P_{l}\left(\mathbf{T}^{a}\right)=P_{l-1}\left(P\left(\mathbf{T}^{a}\right)\right)$. Next, consider the $k$ algebra homomorphism $\Psi^{\prime}: k[\mathbf{T}, \mathbf{U}] \rightarrow k\left[f_{1}, \ldots, f_{r}, \Phi\left(f_{1}\right), \ldots, \Phi\left(f_{r}\right)\right]$ such that $\Psi^{\prime}\left(T_{j}\right)=f_{j}, \Psi^{\prime}\left(U_{j}\right)=\Phi\left(f_{j}\right)$, and let $\Psi$ denote the restriction of $\Psi^{\prime}$ to $k[\mathbf{T}]$.

Let $F(\mathbf{T})=\sum_{a} \alpha_{a} \mathbf{T}^{a} \in k[\mathbf{T}]$ be a homogeneous polynomial of degree $t$, with $a=\left(a_{1}, \ldots, a_{r}\right),|a|=t$ and $\mathbf{T}^{a}=T_{1}^{a_{1}} \cdots T_{r}^{a_{r}}$, and let $s$ denote the common degree of the $f$ 's. We claim that $X_{1}^{s} \Psi^{\prime}(P(F(\mathbf{T}))) \equiv t Y_{1}^{s} \Psi(F(\mathbf{T}))\left(\bmod I_{2}(\mathbf{X}, \mathbf{Y})\right)$. Indeed, it follows from Lemma (2.2.1) that, for a given term $\alpha_{a} \mathbf{T}^{a}$ of $F(\mathbf{T})\left(\alpha_{a} \neq 0\right)$, one has

$$
\Psi^{\prime}\left(P\left(\mathbf{T}^{a}\right)\right) \equiv\left(a_{i(a)}+\ldots+a_{r}\right) \Phi\left(f_{i(a)}\right) f_{i(a)}^{a_{i(a)}-1} f_{i(a)+1}^{a_{i(a)+1}} \cdots f_{r}^{a_{r}},
$$

where $a_{i(a)} \neq 0, a_{i}=0(i<i(a))\left(\bmod I_{2}(\mathbf{X}, \mathbf{Y})\right)$. By summing up over all terms of $F(\mathbf{T})$, one obtains

$$
\Psi(P(F(\mathbf{T}))) \equiv t \sum_{a} \alpha_{a} \Phi\left(f_{i(a)}\right) f_{i(a)}^{a_{i(a)}-1} f_{i(a)+1}^{a_{i(a)+1}} \cdots f_{r}^{a_{r}} \quad\left(\bmod I_{2}(\mathbf{X}, \mathbf{Y})\right) .
$$

Again by Lemma (2.2.1), one has $X_{1}^{s} \Phi\left(f_{j}\right)=Y_{1}^{s} f_{j}$. Substituting yields the desired result.

Next, by iterating the polarization, one easily gets

$$
X_{1}^{l s} \Psi^{\prime}\left(P_{l}(F(\mathbf{T}))\right) \equiv \frac{t !}{(t-l) !} Y_{1}^{l s} \Psi(F(\mathbf{T})) \quad\left(\bmod I_{2}(\mathbf{X}, \mathbf{Y})\right)
$$

where $P_{l}(F(\mathbf{T}))=0$ if $l>t$.

On the other hand, a computation yields

$$
F\left(f_{1} V-\Phi\left(f_{1}\right) U, \ldots, f_{r} V-\Phi\left(f_{r}\right) U\right)=\sum_{l=0}^{t}(-1)^{l} \Psi^{\prime}\left(P_{l}(F(\mathbf{T}))\right) V^{t-l} U^{l} .
$$

Using (3-1) with $l=t$, one gets

$$
X_{1}^{l s} F\left(f_{1} V-\Phi\left(f_{1}\right) U, \ldots, f_{r} V-\Phi\left(f_{r}\right) U\right) \equiv g \Psi(F(\mathbf{T})) \quad\left(\bmod I_{2}(\mathbf{X}, \mathbf{Y})\right),
$$


where

$$
g=\sum_{l=0}^{t}(-1)^{l} \frac{t !}{(t-l) !} X_{1}^{(t-l) s} Y_{1}^{l s} V^{t-l} U^{l} \notin I_{2}(\mathbf{X}, \mathbf{Y})[\mathbf{X}, \mathbf{Y}, U, V] .
$$

One concludes that $F\left(f_{1} V-\Phi\left(f_{1}\right) U, \ldots, f_{r} V-\Phi\left(f_{r}\right) U\right) \in I_{2}(\mathbf{X}, \mathbf{Y})[\mathbf{X}, \mathbf{Y}, U, V]$ if and only if $\Psi(F(\mathbf{T})) \in I_{2}(\mathbf{X}, \mathbf{Y})[\mathbf{X}, \mathbf{Y}, U, V] \cap k[\mathbf{X}]=(0)$.

This finishes the proof.

(3.3) Corollary. Notation as in the beginning of the section. Moreover, let $n=2$. Then $\mathcal{F}(\tilde{I})$ is isomorphic to the homogeneous coordinate ring of the duple Veronese model of $\mathbb{P}^{m-1}$. In particular, $\mathcal{F}(\tilde{I})$ is normal and Cohen-Macaulay.

Proof. By Proposition $(2.2 .3), \mathcal{F}(\tilde{I}) \simeq k\left[M_{\alpha}\right]$, where $M_{\alpha}$ runs through the monomials of degree $d$ in the variables $\mathbf{X}$.

\section{REFERENCES}

[BV] W. Bruns and U. Vetter, Determinantal rings, Lecture Notes in Mathematics, Vol. 1327, Springer Verlag, Berlin-Heidelberg-New York, 1988. MR 89i:13001

[EiSt] D. Eisenbud and B. Sturmfels, Binomial Ideals, Duke Math. J. (to appear).

[Gim] Ph. Gimenez, Étude de la fibre spéciale de l'éclatement d'une variété monomiale en codimension deux, Thèse, Institut Fourier, 1993.

[HSV] J. Herzog, A. Simis and W. V. Vasconcelos, Koszul homology and blowing-up rings, Commutative Algebra, Lecture Notes in Pure and Applied Math., vol. 84, Marcel-Dekker, New York, 1983, pp. 79-169. MR 84k:13015

[Hu] C. Huneke, Powers of ideals generated by weak d-sequences, J. Algebra 68 (1981), 471509. MR 82k:13003

$[\mathrm{HuHu}] \mathrm{S}$. Huckaba and C. Huneke, Powers of ideals having small analytic deviation, Amer. J. Math. 114 (1992), 367-403. MR 93g:13002

[HUT] J. Herzog, N. V. Trung and B. Ulrich, On the multiplicity of blow-ups rings of ideals generated by d-sequences, J. Pure and Appl. Algebra 80 (1992), 273-297. MR 93h:13004

[MoSi] M. Morales and A. Simis, Symbolic powers of monomial curves in $\mathbb{P}^{3}$ lying on a quadric surface, Comm. in Algebra 20(4) (1992), 1109-1122. MR 93c:13005

[Sch] P. Schenzel, Filtrations and noetherian symbolic blowup rings, Proc. Amer. Math. Soc. 102 (1988), 817-822. MR 89b:13029

[Stu] B. Sturmfels, Gröbner bases and Stanley decompositions of determinantal rings, Math. Z. 205 (1990), 137-144. MR 91m:14076

[STV] A. Simis, N. V. Trung and G. Valla, The diagonal subalgebra of a blowup algebra, J. Pure Appl. Algebra (to appear). BRAZIL

imeCC, Universidade Estadual de Campinas, 13081-970 Campinas, São Paulo,

E-mail address: brumatti@ime.unicamp.br

Departamento de Algebra, Geometria e Topologia, Facultad de Ciencias, UniversiDAD DE VAlladolid, 47005 Valladolid, Spain

E-mail address: pgimenez@cpd.uva.es

Universidade Federal da Bahia, Instituto de Matemática, Av. Ademar de Barros, S/N, 40170-210 Salvador, Bahia, BraziL

E-mail address: aron@ufba.br 